\title{
Reconstruction of a large defect of the female chest following keloid excision with use of the rectus abdominis myocutaneous flap
}

\author{
Quan-Cang Men ${ }^{1 *}$, Shu Liu ${ }^{2 *}$, Ke-Xin Song ${ }^{1}$, You-Bin Wang ${ }^{1}$, Zhu Lin ${ }^{1}$, Liu Hao ${ }^{1}$, Xin-Hang Dong ${ }^{1}$, Long Xiao ${ }^{1}$ \\ ${ }^{1}$ Department of Plastic Surgery, Peking Union Medical College Hospital, Beijing 100032, China. \\ ${ }^{2}$ China Meitan General Hospital Affiliated to North China Universityof Science and Technology, Beijing 100028, China. \\ *Authors contributed equally.
}

Correspondence to: Dr. You-Bin Wang, Department of Plastic Surgery, Peking Union Medical College Hospital, Beijing 100032, China.

E-mail:wybenz@sina.com

How to cite this article: Men QC, Liu S, Song KX, Wang YB, Lin Z, Hao L, Dong XH, Xiao L. Reconstruction of a large defect of the female chest following keloid excision with use of the rectus abdominis myocutaneous flap. Plast Aesthet Res 2017;4:86-91.

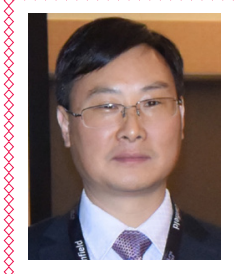

Dr. You-Bin Wang is an outstanding Professor in the Department of Plastic Surgery at Peking Union Medical College Hospital. He is famous in keloid treatment and study. He has invented many new surgical methods in keloid treatment and has published many articles in this field. He is also good at cleft lip surgery, nose reconstructive surgery and breast reconstruction.

\section{Article history:}

Received: 21-03-2017

Accepted: 20-04-2017

Published: 26-05-2017

Key words:

Keloid,

rectus abdominis myocutaneous flap, radiotherapy

\begin{abstract}
Aim: This study aimed to investigate the efficacy of the myocutaneous flap of the rectus abdominis in the surgical treatment of a large defect on the female chest following keloid excision. Methods: According to the location and size of the keloid on the chest, a myocutaneous flap based on the left or right rectus abdominis muscle was designed and transferred for repair of a chest defect following keloid resection. Radiotherapy was performed in the surgical area on the first and seventh postoperative days. Results: From January 2015 to March 2016, rectus abdominis myocutaneous flap coverage and early radiotherapy were used to treat 7 cases of keloids on the female chest. A postoperative follow-up of 10-14 months (average 12 months) was conducted. All the flaps survived well without evidence of keloid recurrence, and all patients achieved an improved chest shape. Conclusion: The rectus abdominis myocutaneous flap is a viablemethod for wound closure following resection of large keloids on the female chest.
\end{abstract}

\section{INTRODUCTION}

Keloids formation occurs secondary to excessive local fibroblast proliferation and synthesis of collagen fibers: ${ }^{[1]}$ this process frequently occurs in the chest wall, shoulder, upper arm and other locations. The disease course of a keloid on the chest wall is longer, and these areas tend to be wider, particularly in

(i) (2) This is an open access article distributed under the terms of the Creative Commons AttributionEY NO SA NonCommercial-ShareAlike 3.0 License, which allows others to remix, tweak, and build upon the work non-commercially, as long as the author is credited and the new creations are licensed under the identical terms.

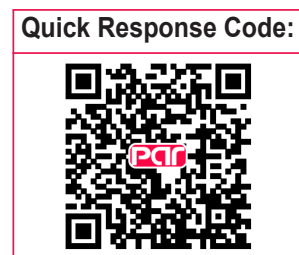


females ${ }^{[2]}$ in whom the lesions frequently involve the breast and other peripheral organs. Many treatment methods have been reported including triple therapy in which surgery is combined with steroid injections and silicone sheet application. ${ }^{[3]}$ While surgery combined with radiotherapy is effective in the treatment of keloids ${ }^{[4]}$ the wound cannot be directly closed following resection of keloids greater than $10 \mathrm{~cm} \times 10 \mathrm{~cm}$ in width and length on the chest wall. In women, given the presence of breast tissue, it can be even more difficult to employ primary closure. Skin grafting would delay radiotherapy. ${ }^{[5]}$ Therefore, for large defects following keloid excision in females a rectus abdominis myocutaneous flap was used to repair the wound, and achieving a better therapeutic efficacy than a skin graft.

\section{METHODS}

Clinical data: 7 female patients, aged between 25 and 46 years, with an average age of $33.40 \pm 8.32$ years presented with keloids of the chest. Five patients had keloid formation following hyperplasia of chest acne infections, and 2 patients developed keloids after the resection of a skin mass. None of the patients had a history of upper abdominal surgery.

\section{Preoperative preparation}

Large keloids frequently have surface irregularities and crevices which can permit the accumulation of contaminating substances and put the patient at risk for postoperative infection. In cases in which an infection is already present, debridement, drainage and dressings should be performed until the infection has been brought under control. Three days prior to surgery, daily povidone-iodine disinfection was performed for patients who have recently recovered from an infection or in whom sinuses have formed beneath the keloid mass. All other patients were required to shower daily beginning three days prior to surgery. With instructions to thoroughly clean the keloid and any depressed region or gaps. Cotton swabs were used during each cleanse and running water was used to rinse the gaps.

The skin surrounding the keloid mass was also determined to be healthy and clean prior to surgery. In cases in which infective acne was present in the surrounding skin, daily $75 \%$ alcohol local disinfection was performed until the infection was controlled. For cases in which the acne was in its recovery stage with resolution of erythema and pain, the remaining pustules were cleaned and the wounds were covered with sterile gauze. Patients were healthy and received medical clearance prior to surgery. Blood glucose levels were controlled in diabetic patients. Prior to beginning surgery, an abdominal flap was designed based on

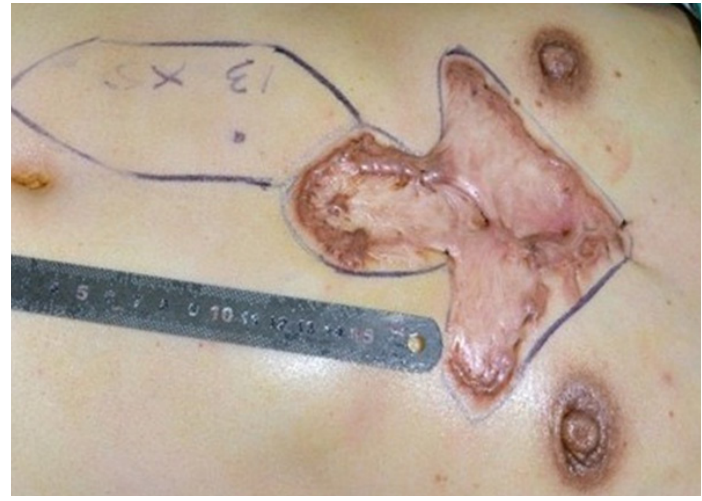

Figure 1: Keloid on the chest and flap design

the size of the keloid. A Doppler stethoscope was first used to determine the site of perforation of the superior epigastric artery into the upper abdominal skin, and then, according to the perforation point, the rotation point of the flap pedicle, the size of the flap, and the location of the flap were determined and marked [Figure 1].

\section{Surgical methods}

The operation was performed under general anesthesia. The patient was placed in the supine position, and the chest and abdomen were disinfected and covered with sterile drapes. Local infiltration anesthesia was performed using a $0.06 \%$ lidocaine $(1: 20,000$ adrenaline) solution in the area surrounding the planned incisions. An incision extending to the deep fascia was first created around the keloid on the chest. The keloid tissue was then removed at the level of the deep fascia. Complete hemostasis was performed. For those patients in whom a preoperative local infection and sinus had been present, the wound was rinsed with hydrogen peroxide solution followed by saline. For patients without a pre-existing infection, saline solution alone was used for irrigation. After re-confirming the size and shape of the wound as well as the design of the abdominal flap, an incision was made within the markings of the designed flap and extended to the deep fascia. The anterior sheath of the rectus abdominis was separated and opened. The rectus abdominis muscle was exposed and separated from its posterior sheath. The rectus abdominis was divided distally with vessel ligation. The flap was elevated proximally to the level of the xiphoid until the flap could freely rotate to the chest using the superior epigastric artery as the pedicle [Figures 2 and 3]. Following complete hemostasis, the flap was transferred to cover the chest wound and the abdominal donor site wound was closed [Figure 4]. As much of the anterior rectus sheath was preserved as possible in order to maintain the integrity of the abdominal wall.

\section{Postoperative treatment}

Radiotherapy was administered to the chest and 


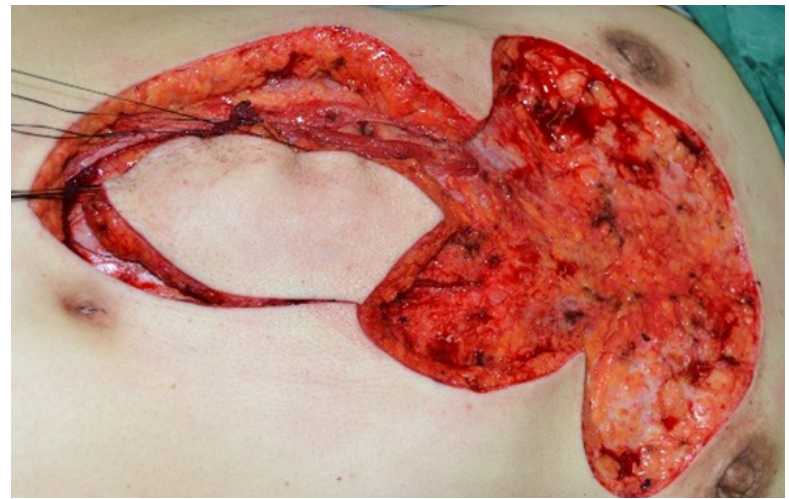

Figure 2: After the formation of the myocutaneous flap of the rectus abdominis

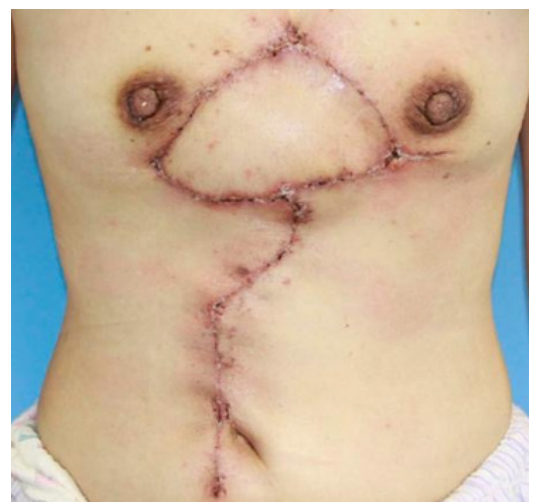

Figure 4: Closed chest wound 2 weeks after flap transfer

abdominal donor site on the first and seventh postoperative days; each dose was 900 cGy for a total dose of 1,800 cGy. The wound was checked and cleaned every three days following surgery and sutures were removed 14 days postoperatively. A compression garment was used 1 month after suture removing until the wound scar become pale and flat.

\section{RESULTS}

From January 2015 to March 2016, the rectus abdominis myocutaneous flap was used combined

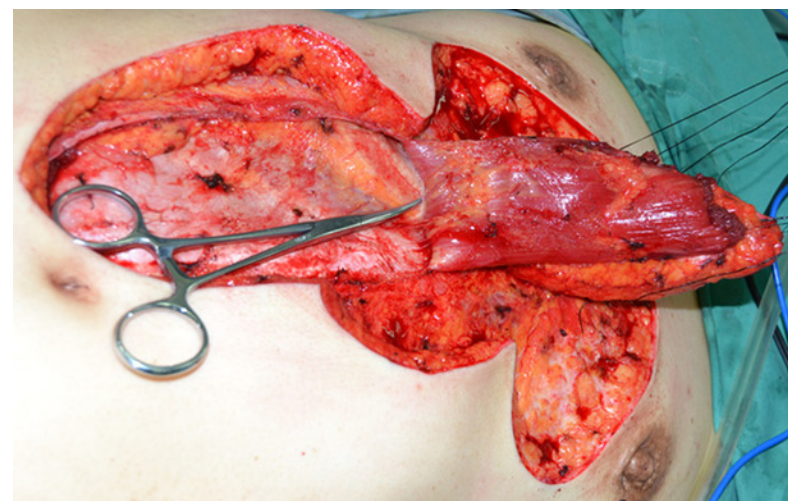

Figure 3: The pedicle with the perforating vessels of the superior epigastric artery

with early radiotherapy to treat 7 cases of large keloids on the female chest. All flaps survived, and the incisions healed during the primary stage without any cases of infection, dehiscence or other complications. All patients completed their courses of thoracic and abdominal radiotherapy. Follow-up was conducted for 10-14 months (average of 12 months) and demonstrated that all incisions healed well without any cases of keloid recurrence. Furthermore, the patients were satisfied with the shape of the chest and abdomen [Figures 5 and 6].

\section{DISCUSSION}

Keloids occur in the skin and can expand rapidly towards surrounding normal tissue. Keloids are pathologically composed of collagen fibers and often protrude clinically from the skin. They often occur epidemiologically in young people, especially in females. ${ }^{[6,7]}$ Keloids frequently form on the chest, shoulder, and lower mandible of the face secondary to acne.

Various therapeutic management techniques have been reported in literature including conventional surgery, cryosurgery, medical therapy including steroid and
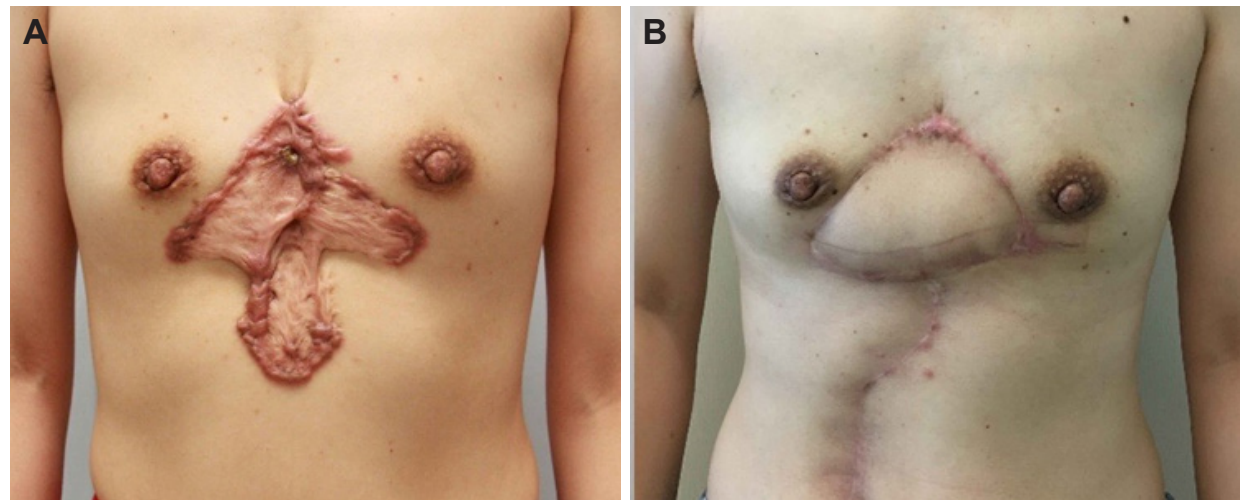

Figure 5: Case 1. (A) Preoperative chest keloid and (B) 8 months after the keloid resection and the repair with the rectus abdominis myocutaneous flap 

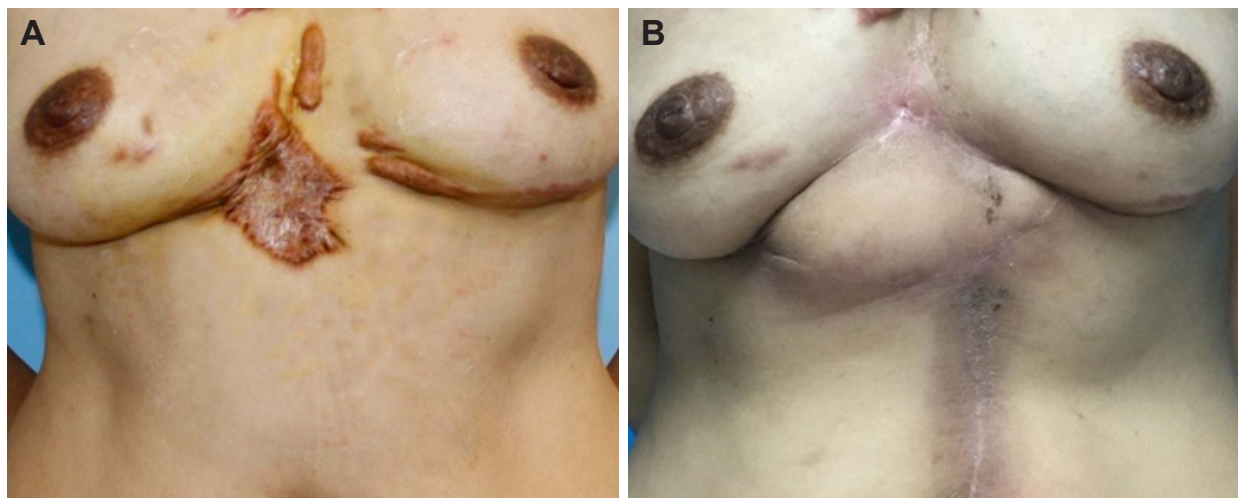

Figure 6: Case 2. (A) Preoperative chest keloid and (B) 1 year after the keloid resection and the repair with the rectus abdominis myocutaneous flap

5-flourouracil injections, and physical modalities such as radiation and laser treatment. Combination usage of these techniques has also been widely reported. 5 -flourouracil combined with steroid injections is often used with clinical results better than with 5-flourouracil alone. ${ }^{[8]}$ Laser treatments in conjunction with steroid injections has also been reported..$^{[9]}$ Careta et al. ${ }^{[10]}$ reported encouraging results following the use of cryosurgery with intralesional steroid injections in the treatment of earlobe keloids. However, the combination of surgery with other techniques is more widely used, particularly the combination of surgery and radiotherapy. Mankowski et al. ${ }^{[11]}$ suggested that surgery and postoperative radiotherapy was the most effective method out of all keloid treatment modalities.

The surgical resection of scar tissue combined with postoperative radiotherapy can inhibit the proliferation of fibroblasts and the synthesis of collagen proteins during early wound healing. Postoperative radiotherapy is a reliable method for the treatment of keloids. The primary requirement for early postoperative radiotherapy is wound closure following resection of the keloid; otherwise, radiotherapy cannot be applied. Primary closure is strongly recommended in this method. ${ }^{[12]}$

Methods for wound closure include direct closure, local flap transfer, internal mammary artery (IMA) perforator flap transfer, and skin grafting. ${ }^{[13-16]}$ Different methods can be selected based on the width of the keloid on the anterior chest wall. For single or multiple isolated lesions with a diameter within $1-3 \mathrm{~cm}$, keloid resection and direct closure can be performed. If direct closure cannot be readily achieved, skin flaps or skin grafts should be performed to decrease the wound tension. For chest wall lesions with a diameter greater than $3 \mathrm{~cm}$, a local flap or IMA perforator flap can be performed to cover the defect and avoid wound dehiscence. For lesions less than $5 \mathrm{~cm} \times 5 \mathrm{~cm}$, randomized local flaps are possible with primary closure of the donor site. IMA perforator flaps are a better option for keloids larger than $5-10 \mathrm{~cm}$ in diameter. For very large keloids larger than $10 \mathrm{~cm} \times 10 \mathrm{~cm}$, skin grafts are the best option following resection. ${ }^{[5]}$

Although both primary closure and local flap transfer are suitable for the surgical treatment of small keloids, free flaps can more adequately cover larger wounds. There are particular challenges associated with wound coverage of large chest keloids in female patients secondary to aesthetic considerations of the breasts. The wound can be covered with a skin graft, but layers of gauze covering the skin graft region often affect the performance and efficacy of radiotherapy. Conversely, radiotherapy may also cause a failure of the skin graft. In addition, for female patients, the breasts limit the effective use of a chest flap. Therefore, choosing an appropriate wound repair method is both challenging and important in the treatment of large thoracic keloids in females. The blood supply to the rectus abdominis myocutaneous flap is reliable with minimal injury to the donor site. ${ }^{[17]}$ For wounds following resection of a keloid on the chest wall, and especially on the distal aspect, the rectus abdominis muscle flap not only is easy to transfer but provides a good tissue match to therecipientsite in color, texture and thickness. ${ }^{[18]}$

The rectus abdominis muscle is located on both sides of the median line of the anterior abdominal wall of the human body, and has a relatively constant blood supply from the superior and inferior epigastric arteries. The superior epigastric artery generates a thick perforating artery at the proximal aspect of the rectus abdominis muscle. The external diameter of this perforating vessel is often larger than $1 \mathrm{~mm}$. The perforating branches are primarily distributed within a range of one tendinous insetion above the umbilicus and $8.0 \mathrm{~cm}$ below the umbilicus. The medial perforating vessels are often the dominant blood vessels among the perforating vessels of the inferior epigastric artery. ${ }^{[19]}$ Because the superior and inferior epigastric arteries have a relatively wide 
range of distribution of perforating vessels to the abdominal skin, specific perforating vessels can be selected according to the size of the chest wound and its distance from the abdomen.

The rectus abdominis muscle is important in maintaining abdominal wall strength. Partial preservation of this muscle therefore essential when designing the rectus abdominis myocutaneous flap. In a report by Chen et al., ${ }^{[20]} 8$ patients underwent breast reconstruction with the muscle-sparing TRAM flap. Of these 8 patients, 2 experienced borderline necrosis and subcutaneous fat liquefaction within zone IV of the flap, with healing following debridement. Although partial skin flap necrosis is well-tolerated in breast reconstruction patients, it is problematic in keloid treatment as it delays the initiation of postoperative radiotherapy. This scenario also increases the risk for keloid recurrence secondary to chronic inflammation. ${ }^{[21]}$ Abdominal wall strength is preserved and herniation is prevented by saving the anterior sheath of the rectus abdominis muscle.

The IMA perforator flap is another option for patients with keloids of the chest wall. Trauma to the abdominal wall is minimized with this method as compared to the rectus abdominis myocutaneous flap. Ogawa et al. ${ }^{[22]}$ have reported use of this skin flap design in chest wall keloid wound coverage. In their report, a skin flap measuring $14 \mathrm{~cm} \times 5 \mathrm{~cm}$ was designed based on the dominant perforators of the IMA in the 6th, 7th, 8th and 9th intercostal spaces. In the current study, however, the borders of the keloids extended more distally. The perforators were difficult to identify by Doppler ultrasound secondary to the overlying keloid mass. These perforators would also have been damaged following resection of the, and therefore would have been unsuitable for use as a flap pedicle. The rectus abdominis myocutaneous flap is a more appropriate choice in this situation for very large keloids which cover the inferior chest and upper abdominal wall.

Although use of the rectus abdominis myocutaneous flap from the abdominal wall for wound repair following keloid resection can provide a reliable blood supply and good soft tissue match, the extent of surgical trauma and the patients' medical status demands that one consider the following points when selecting this surgical method:

(1) This surgical approach is appropriate for women and patients engaged in light physical labor because of the effect of muscle harvest on abdominal wall strength. For men and patients engaged in moderate to heavy physical labor, the method should be selected only after careful consideration. During surgery, the integrity of one side of the rectus abdominis muscle is compromised, which to a certain degree damages the strength and integrity of the abdominal wall. Therefore, for young male keloid patients, this surgical method is not recommended, and is why all patients in this series were women.

(2) The rectus abdominis muscle flap is more suitable for wound recovery following resection of a keloid located in the middle or distal aspect of the chest. Secondary to limitations of the length of the pedicle, it would be difficult to repair a keloid located in the middle or proximal chest. Moreover, the subcutaneous tissue of the flap is quite thick which provides a poor match to tissue of the upper chest. In addition, the longer the pedicle required, the wider the dissection must be, increasing the size of the donor site defect. For patients who have demonstrated a propensity towards keloid formation, the risk of postoperative scar hyperplasia or additional keloid formation is even greater. When a keloid in the middle or lower part of the chest wall has been repaired with a myocutaneous flap from the rectus abdominis muscle in the middle or upper abdomen, the postoperative incision will be located within the radiotherapy range, which is both convenient for performing radiotherapy and reduces the extent of radiation damage to the normal tissue. Therefore, it is recommended that a myocutaneous flap of the rectus abdominis from the middle or upper abdomen be used to repair the wound following keloid resection from the middle or lower chest.

(3) Although a rectus abdominis myocutaneous flap can provide a large portion of tissue for wound repair, the amount of tissue that this approach can provide is nevertheless limited. When the wound following resection exceeds the amount of tissue that can be provided by the flap, one must additionally consider other methods or the use of multiple flaps in combination for repair.

\section{Authors' contributions}

Manuscript's preparation: Q.C. Men, S. Liu Manuscript's review: Y.B. Wang, Z. Lin, L. Xiao Concept design: Y.B. Wang

Literature search: K.X. Song, L. Hao, X.H. Dong

\section{Financial support and sponsorship}

None.

\section{Conflicts of interest}

There are no conflicts of interest.

\section{Patient consent}

Informed consent was obtained from the patients. 


\section{Ethics approval}

All treatment procedures were provided in accordance with the ethics approval of Peking Union Medical College Hospital.

\section{REFERENCES}

1. Jin J, Gao JH. Research development in the pathogenic mechanism of keloids. J Clin Rehabil Tissue Eng Res 2007;11:1953-6,73.

2. Kong BY, Baek GH, Gong HS. Treatment of keloid formation following syndactyly division: surgical technique. Hand Surg 2012;17:433-7.

3. De Sousa RF, Chakravarty B, Sharma A, Parwaz MA, Malik A. Efficacy of triple therapy in auricular keloid. J Cutan Aesthet Surg 2014;7:98-102.

4. Flickinger JC. A radiobiological analysis of multicenter data for postoperative keloid radiotherapy. Int $J$ Radiat Oncol Biol Phys 2011;79:1164-70.

5. Long X, Zhang M, Wang Y, Zhao R, Wang Y, Wang X. Algorithm of chest wall keloid treatment. Medicine (Baltimore) 2016;95:e4684.

6. Lu WS, Zheng XD, Yao XH, Zhang LF. Clinical and epidemiological nanlysis of keloid in Chinese patients. Arch Dermatol Res 2015;307:109-14.

7. Sun LM, Wang KH, Lee YC. Keloid incidence in Asian people and its comorbidity with other fibrosis-related diseases: a nationwide population-based study. Arch Dermatol Res 2014;306:803-8.

8. Bijlard E, Steltenpool S, Niessen FB. Intralesional 5-fluorouracil in keloid treatment: a systematic review. Acta Derm Venereol 2015;95:778-82

9. Martin MS, Collawn SS. Combination treatment of $\mathrm{CO} 2$ fractional laser, pulsed dye laser, and triamcinolone acetonide injection for refractory keloid scars on the upper back. J Cosmet Laser Ther 2013;15:166-70

10. Careta MF, Fortes AC, Messina MC, Maruta CW. Combined treatment of earlobe keloids with shaving, cryosurgery, and intralesional steroid injection: a 1-year follow-up. Dermatol Surg 2013;39:734-8.

11. Mankowski P, Kanevsky J, Tomlinson J, Dyachenko A, Luc M.
Optimizing radiotherapy for keloids: a meta-analysis systematic review comparing recurrence rates between different radiation modalities. Ann Plast Surg 2017;78:403-11.

12. van Leeuwen MC, Stokmans SC, Bulstra AE, Meijer OW, Heymans MW, Ket JC, Ritt MJ, van Leeuwen PA, Niessen FB. Surgical excision with adjuvant irradiation for treatment of keloid scars: a systematic review. Plast Reconstr Surg Glob Open 2015;3:e440.

13. Wang YB, Long X. Double layer continuous intradermal sutures in keloid operation. J Clin Exp Dermatol Res 2014;5:205.

14. Xue D, Qian H. Surgical management for large chest keloids with internal mammary artery perforator flap. An Bras Dermatol 2016;91:103-5.

15. Berman B, Maderal A, Raphael B. Keloids and hypertrophic scars: pathophysiology, classification, and treatment. Dermatol Surg 2017;43 Suppl 1:S3-18.

16. Akita S, Akino K, Yakabe A, Imaizumi T, Tanaka K, Anraku K, Yano $\mathrm{H}$, Hirano A. Combined surgical excision and radiation therapy for keloid treatment. J Craniofac Surg 2007;18:1164-9.

17. Sun JM, Kua JE, Lee LS, Leo KW. The oblique rectus abdominis musculocutaneous flap for reconstruction after resection of a spermatic cord liposarcoma. Arch Plast Surg 2015;42:647-50.

18. Deo SV, Nootan KS, Niranjan B, Dinesh K. Vertical rectus abdominis myocutaneous flap cover for lower abdomen, chest wall, groin and thigh defects following resection of malignant tumours. Indian $J$ Cancer 2001;38:33-7.

19. Holm C, Mayr M, Höfter E, Ninkovic M. Perfusion zones of the DIEP flap revisited: a clinical study. Plast Reconstr Surg 2006;117:37-43.

20. Chen XJ, Wang B, Zheng HB, Shan XY, Zhang HH, Wang MS, Zhang DJ, Zhuang EL. Anatomic study and application of TRAM flap with partial preservation of abdominal rectus muscle in the breast reconstruction. Zhonghua Zheng Xing Wai Ke Za Zhi 2012;28:248-52.

21. Lee KW, Burm JS, Yang WY. Keloid formation on the great toe after chronic paronychia secondary to ingrown nail. Int Wound $J$ 2013;10:200-2.

22. Ogawa R, Ono S, Akaishi S, Dohi T, Iimura T, Nakao J. Reconstruction after anterior chest wall keloid resection using internal mammary artery perforator propeller flaps. Plast Reconstr Surg Glob Open 2016;4:e1049. 